Louisiana State University

LSU Digital Commons

$1-1-1999$

\title{
Controlling phase matching of high-order harmonic generation by manipulating the fundamental field
}

\author{
Lena Roos \\ Lunds Tekniska Högskola \\ Eric Constant \\ Université de Bordeaux \\ Eric Mével \\ Université de Bordeaux \\ Philippe Balcou \\ CNRS Centre National de la Recherche Scientifique \\ Dominique Descamps \\ Lunds Tekniska Högskola
}

See next page for additional authors

Follow this and additional works at: https://digitalcommons.Isu.edu/physics_astronomy_pubs

\section{Recommended Citation}

Roos, L., Constant, E., Mével, E., Balcou, P., Descamps, D., Gaarde, M., Valette, A., Haroutunian, R., \& L'Huillier, A. (1999). Controlling phase matching of high-order harmonic generation by manipulating the fundamental field. Physical Review A - Atomic, Molecular, and Optical Physics, 60 (6), 5010-5018. https://doi.org/10.1103/PhysRevA.60.5010

This Article is brought to you for free and open access by the Department of Physics \& Astronomy at LSU Digital Commons. It has been accepted for inclusion in Faculty Publications by an authorized administrator of LSU Digital Commons. For more information, please contact ir@lsu.edu. 


\section{Authors}

Lena Roos, Eric Constant, Eric Mével, Philippe Balcou, Dominique Descamps, Mette B. Gaarde, Alexandre Valette, Romain Haroutunian, and Anne L'Huillier 


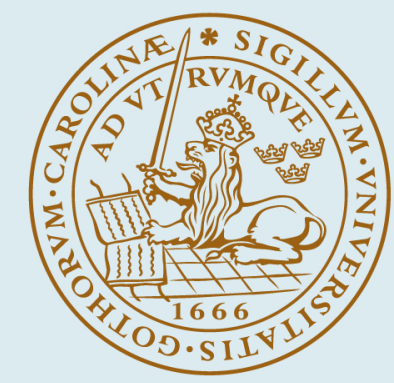

\title{
LUND UNIVERSITY
}

\section{Controlling phase matching of high-order harmonic generation by manipulating the fundamental field}

\author{
Roos, L; Constant, E; Mevel, E; Balcou, P; Descamps, D; Gaarde, M. B; Valette, A; \\ Haroutunian, R; L'Huillier, Anne \\ Published in: \\ Physical Review A (Atomic, Molecular and Optical Physics)
}

DOI:

10.1103/PhysRevA.60.5010

1999

\section{Link to publication}

Citation for published version (APA):

Roos, L., Constant, E., Mevel, E., Balcou, P., Descamps, D., Gaarde, M. B., Valette, A., Haroutunian, R., \& L'Huillier, A. (1999). Controlling phase matching of high-order harmonic generation by manipulating the fundamental field. Physical Review A (Atomic, Molecular and Optical Physics), 60(6), 5010-5018. https://doi.org/10.1103/PhysRevA.60.5010

Total number of authors:

9

\footnotetext{
General rights

Unless other specific re-use rights are stated the following general rights apply:

Copyright and moral rights for the publications made accessible in the public portal are retained by the authors and/or other copyright owners and it is a condition of accessing publications that users recognise and abide by the legal requirements associated with these rights.

- Users may download and print one copy of any publication from the public portal for the purpose of private study or research.

- You may not further distribute the material or use it for any profit-making activity or commercial gain

- You may freely distribute the URL identifying the publication in the public portal

Read more about Creative commons licenses: https://creativecommons.org/licenses/

Take down policy

If you believe that this document breaches copyright please contact us providing details, and we will remove access to the work immediately and investigate your claim.
} 


\title{
Controlling phase matching of high-order harmonic generation by manipulating the fundamental field
}

\author{
Lena Roos, ${ }^{1}$ Eric Constant, ${ }^{2}$ Eric Mével, ${ }^{2}$ Philippe Balcou, ${ }^{3}$ Dominique Descamps, ${ }^{1}$ Mette B. Gaarde, ${ }^{1,4}$ Alexandre Valette, ${ }^{3}$ \\ Romain Haroutunian, ${ }^{3}$ and Anne L'Huillier ${ }^{1}$ \\ ${ }^{1}$ Department of Physics, Lund Institute of Technology, P.O. Box 118, S-221 00 Lund, Sweden \\ ${ }^{2}$ CELIA/CPMOH, Université Bordeaux 1, Cours de la Libération, F-33405 Talence Cedex, France \\ ${ }^{3}$ Laboratoire d'Optique Appliquée, ENSTA-Ecole Polytechnique, Unité Mixte de Recherches 7639, Centre National de la Recherche \\ Scientifique, F-91761 Palaiseau Cedex, France \\ ${ }^{4}$ Niels Bohr Institute, Orsted Laboratory, 2100 Copenhagen, Denmark
}

(Received 1 July 1999)

\begin{abstract}
We study experimentally how to control and improve phase matching of high-order harmonic generation. We use a birefringent lens and a birefringent compensator to obtain a fundamental laser pulse $(150 \mathrm{fs}, 800 \mathrm{~nm}$, $\sim 4 \mathrm{~mJ}$ ) with two foci separated by $6.2 \mathrm{~mm}$ along the propagation axis and with a controllable phase delay between the polarizations along the optic axes of the birefringent optical components. This enables us to enhance the high-order harmonic conversion efficiency for the high-order harmonics in neon to $3 \times 10^{-8}$, a factor of 4 higher compared to a single-focus setup in similar conditions. The enhancement is achieved by improving the phase matching and at the same time maintaining a high intensity in a large generating volume. [S1050-2947(99)04512-6]
\end{abstract}

PACS number(s): 42.65.Ky, 32.80.Rm

\section{INTRODUCTION}

In recent years, high-order harmonic generation has become a promising source of short-pulse coherent radiation in the extreme ultraviolet (XUV) range. A large effort is devoted to increase its photon number. Early studies have mostly concentrated on optimization with respect to atomic or molecular gases and to the laser wavelength, thus addressing the single-atom emission. The influence of the focusing geometry, as well as of the position of the gas medium relative to the laser focus, on the phase matching of high-order harmonics has been pointed out $[1,2]$. Recently, several groups have studied harmonic generation using ultrashort laser pulses focused onto a hollow fiber, [3-5] reporting high conversion efficiencies. These results, however, only concern heavy rare gases, xenon and argon. Tamaki and co-workers [6] report a very high conversion efficiency in neon, and attribute it to the formation of a filament in the laser focal region. They also point out the importance of controlling the intrinsic phase of the harmonics to optimize the efficiency.

In the present paper, we manipulate the amplitude and the phase of the fundamental field in order to improve the phase matching of high-order harmonics. We use neon, for which dispersion effects are not as important as for heavier noble gases. The idea is to get a large generating volume, where the intensity is high and where both the intensity profile and the phase variation of the fundamental field are as flat as possible. To this end, we use birefringent optical components, a lens and a compensator, in order to get two field polarizations focused at two different places along the propagation axis. The phase difference between the two components can be continuously varied. The optical components were used in a previous experiment to manipulate the polarization of the fundamental in space and consequently the spatial profile of the harmonics [7]. We interpret our experi- mental results by an analysis of phase matching both along the propagation axis and in the whole interaction volume.

We first describe the experimental setup in Sec. II. In order to understand the advantages of a two-foci setup and to be able to interpret the experimental results we then discuss the phase matching of high-order harmonics, using simple one- and three-dimensional calculations in Sec. III. The experimental results are presented in Sec. IV. A short summary is given in Sec. V.

\section{EXPERIMENTAL METHOD}

A schematic view of the experimental setup is shown in Fig. 1. The laser is the titanium sapphire terawatt laser at the Lund High-Power Laser Facility [8]. The central laser wavelength is $800 \mathrm{~nm}$, the repetition rate $10 \mathrm{~Hz}$, and the pulse duration $150 \mathrm{fs}$. The main optical components used to gen-

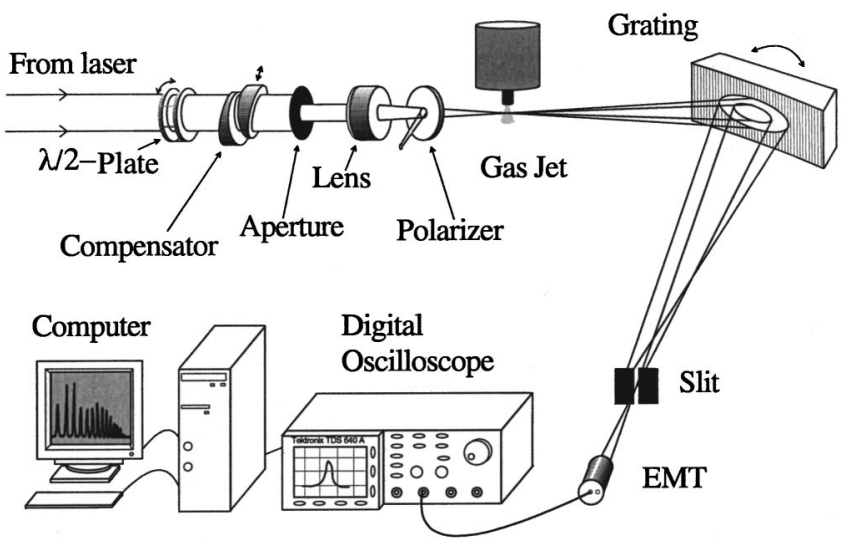

FIG. 1. Experimental setup used for generating a laser pulse that focuses on two separated spots along the propagation axis. Highorder harmonics are generated in a gas jet, dispersed by a grating, and detected by an electron multiplier (EMT). 
erate the two foci are a birefringent compensator, a birefringent lens with an average focal length of $40 \mathrm{~cm}$, and a polarizer. The incoming laser light is linearly polarized in the horizontal plane. The birefringent quartz lens is oriented with its optic axis at an angle of $45^{\circ}$ to the laser polarization. The electrical field of the laser beam is thus split up into two orthogonally polarized components with equal amplitude propagating in the same direction. The two polarizations experience two different refractive indices through the lens, the ordinary and the extraordinary, and therefore focus on two different spots along the propagation axis, separated by 6.2 $\mathrm{mm}$. The lens induces a time separation between the two pulses of $\sim 300$ fs. This time delay is compensated for by two birefringent quartz wedges with their optic axes at a $90^{\circ}$ angle to that of the lens, equivalent to a birefringent plate with controllable thickness. By translating one of the wedges we can also finely tune the time delay and hence the phase difference $(\phi)$ between the two polarizations. A translation of one wedge of $3.1 \mathrm{~mm}$ corresponds to a change in phase difference of $2 \pi$ radians.

After the compensator and the lens, the laser beam has a transversally varying ellipticity [7]. We use a polarizer to select the horizontal polarization (see Fig. 1). This ensures that the total field is linearly polarized in the interaction region.

Since an important task in the experiment consists in comparing the harmonic yield of a fundamental field with two foci to the yield obtained with one focus, we insert a half-wave plate in the path of the beam. By rotating the wave plate we can set the polarization of the beam parallel to the optic axes of the lens and thereby get only one focal point. Note that about half the pulse energy is reflected by the polarizer in both configurations.

The laser pulse is apertured down from a diameter of approximately $40 \mathrm{~mm}$ to a diameter of around $12 \mathrm{~mm}$. The total pulse energy in the interaction region, distributed in one or two foci, is varied between 2 and $5 \mathrm{~mJ}$. The experimental intensities estimated by comparing the cutoff energy in our spectra with the approximate cutoff law $I_{p}+2 U_{p}$ [9], accounting for propagation effects, are around 4-6 $\times 10^{14} \mathrm{~W} / \mathrm{cm}^{2}$ which is sufficient to generate harmonic orders as high as 61 . The harmonics are produced in a pulsed gas jet filled with neon atoms (backing pressure 1.4-4 bars). The length of the gas medium is estimated to be about $1 \mathrm{~mm}$ in the interaction region.

The harmonics are dispersed by a rotating grazingincidence toroidal grating that focuses them onto a $200 \mu \mathrm{m}$ wide slit. The harmonic signal is detected by an electron multiplier (EMT) and recorded by a digital oscilloscope connected to a personal computer (see Fig. 1).

In all the harmonic spectra presented below, the harmonic yields are given in units of the absolute number of emitted photons per bandwidth. The EMT used in the measurements has been calibrated with a photodiode (AXUV-100). As opposed to the EMT, the diode is very sensitive to the fundamental laser light and therefore needs to be shielded with an aluminum filter. We measure the absolute number of photons behind the $200 \mu \mathrm{m}$ slit at a given wavelength $(38.1 \mathrm{~nm})$. To infer the number of emitted harmonic photons from the number of photons detected on the photodiode we account for the losses on the grating (reflectivity and diffraction efficiency) and through the aluminum filter. The error in the estimation of the given number of emitted photons is about a factor of 3 .

The resolution of the spectrometer is approximately 0.05 $\mathrm{nm}$, i.e., much less than the width of a typical harmonic peak. The number of photons in one harmonic can be obtained by integrating its spectrum.

The position of the gas jet can also be varied and its position relative to the foci is given with an uncertainty of about $1 \mathrm{~mm}$.

\section{PHASE MATCHING IN THE TWO-FOCI CONFIGURATION}

In this section, we investigate theoretically how phase matching can be improved by using a beam with two foci instead of one. We first describe how we calculate the fundamental field in the focal region (Sec. III A). To introduce the basic concepts of our method and clarify our idea, we first consider phase matching on the propagation axis only. We compare the two-foci case with the single-focus case. Then we examine the role of the phase difference between the two polarizations propagating along the optic axes of the birefringent optics (Sec. III B). The next step is to consider phase matching in the whole volume where harmonics can be emitted and not just on axis (Sec. III C). Finally, we discuss the effect of dispersion (Sec. III D).

\section{A. Calculation of the fundamental field}

The amplitude of the electrical field of the two orthogonally polarized components right after the compensator, the aperture and the lens (see Fig. 1), $u_{x}$ and $u_{y}$, can be expressed as

$$
u_{x}\left(r_{0}\right)=u_{x}^{0} \exp \left(-\frac{i \pi r_{0}^{2}}{f_{x} \lambda}\right) \exp \left(-\frac{r_{0}^{2}}{w^{2}}\right)
$$

and

$$
u_{y}\left(r_{0}\right)=u_{y}^{0} \exp \left(-\frac{i \pi r_{0}^{2}}{f_{y} \lambda}\right) \exp \left(-\frac{r_{0}^{2}}{w^{2}}\right) \exp (-i \phi) .
$$

This is valid for $r_{0}<a$, where $a$ is the radius of the aperture and $r_{0}$ is the transverse coordinate right after the lens, $u_{x}\left(r_{0}\right), u_{y}\left(r_{0}\right)=0, r_{0}>a$. The first factor in the two equations is the field amplitude at $r_{0}=0, u_{x}^{0}=u_{y}^{0}$. The second factor is the phase due to focusing, for the first component with focal length $f_{x}$ and for the second component with focal length $f_{y}$, where $\lambda$ is the laser wavelength. The third factor is the transverse variation of the field amplitude for a Gaussian beam with spot size $w$. The phase difference between the two orthogonally polarized components is $\phi$. When $\phi=0$ the two fields are in phase on axis right after the lens. $\phi$ is controlled by varying the thickness of the compensator. The total field after the lens is elliptically polarized. The degree of ellipticity, which depends on the phase difference between the two components, varies in space [7], since the focal lengths $f_{x}$ and $f_{y}$ are not equal. In the one-focus case, there is only one field component and no phase difference.

After the polarizer, the beam is linearly polarized and the field amplitude is $u_{0}\left(r_{0}\right)=\left[u_{x}\left(r_{0}\right)+u_{y}\left(r_{0}\right)\right] / \sqrt{2}$. Since the 

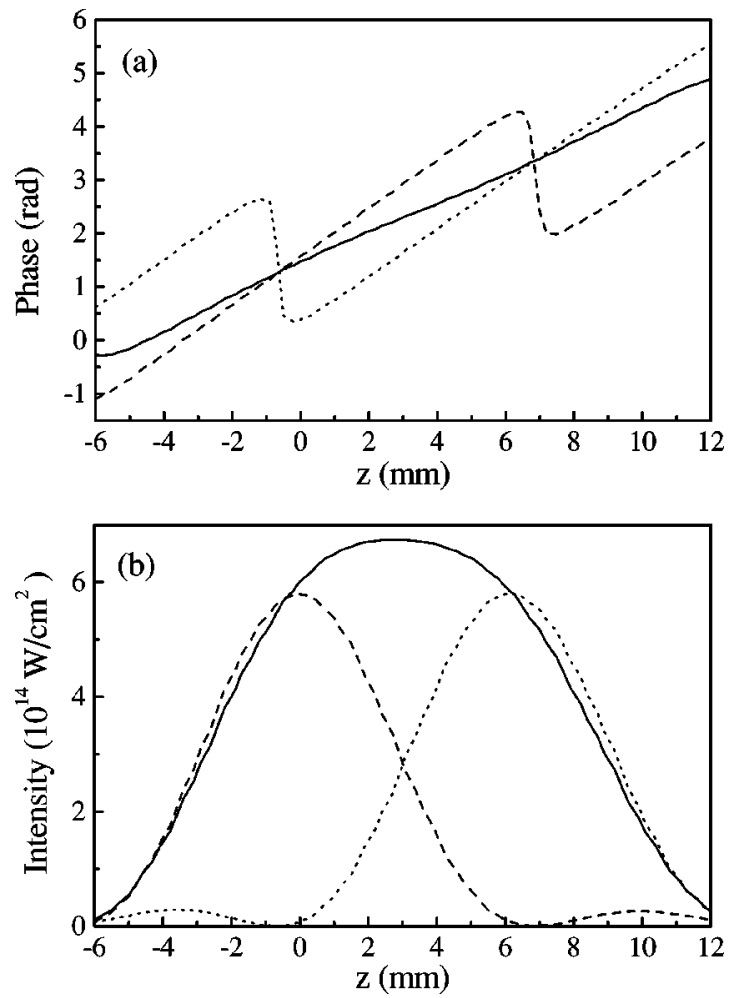

FIG. 2. Comparison of the fundamental field on axis between the configuration with two foci and with one focus. The phase of the field is shown in (a) and the intensity profile in (b). The results for the first focus $(z=0)$ are shown as a dashed line and those for the second as a dotted line $(z=6.2 \mathrm{~mm})$. The intensity and phase for the two-foci case, with a phase difference of $\phi=4.8 \mathrm{rad}$ between the two foci, are shown in solid line.

degree of ellipticity of the beam before the polarizer varies in space, with cylindrical symmetry around the axis of propagation, the intensity of the beam after the polarizer varies in space and presents circular fringes in the plane perpendicular to the propagation axis. The fundamental field in the focal region can be calculated from the electrical field right after the optical components using a Hankel transform [10],

$$
\begin{aligned}
u(r, z)= & \frac{2 \pi i}{\left(f_{y}+z\right) \lambda} \int_{0}^{a} r_{0} u_{0}\left(r_{0}\right) \exp \left(-i \frac{\pi}{\left(f_{y}+z\right) \lambda}\right. \\
& \left.\times\left(r^{2}+r_{0}^{2}\right)\right) J_{0}\left[2 \pi r r_{0} /\left(f_{y}+z\right) \lambda\right] d r_{0},
\end{aligned}
$$

where $r, z$ denote the transverse and longitudinal coordinates in the focal region ( $z=0$ at the first focus), and $J_{0}$ is the zero-order Bessel function.

Note that, even in the one-focus case, the beam does not behave as a Gaussian beam in the focal region. The hard aperture used in the setup induces phase and intensity variations across the focus which are considerably slower than those of a Gaussian beam with a spot size equal to the radius of the aperture. For a Gaussian beam with beam waist $w_{0}$ at $z=0$, the phase shift is $\phi_{\text {Gauss }}=\arctan (2 z / b)$ where $b$ is the confocal parameter $\left(b=2 \pi w_{0}^{2} / \lambda\right)$ and the intensity variation is hyperbolic $\left[I_{\text {Gauss }}=I_{0} /\left(1+4 z^{2} / b^{2}\right)\right]$. For a truncated Gaussian, as is shown below, (see Fig. 2), the intensity variation resembles that of an Airy pattern, with minima on the propagation axis. The phase changes by $\sim 2.3 \mathrm{rad}$ where the intensity has a minimum. (The corresponding shift is $\pi$ for an Airy pattern.)

We have compared the intensities calculated by using Eq. (3), assuming that our laser beam is diffraction limited to the intensities estimated from the cutoff in the experimental spectra. The intensities obtained from calculations are on average 3 times higher than the experimental ones which is probably due to the fact that the laser beam is not diffraction limited. In order to obtain theoretical plots that represent realistically our experimental conditions we use an energy of $1.5 \mathrm{~mJ}$ which is in general lower than those used in the experiments. We stress that the conclusions that can be drawn from our calculations are not very much intensity dependent.

In the following section, we describe the behavior of the intensity and phase of the fundamental beam in the focal region, in the one-focus and two-foci cases.

\section{B. Phase matching on the propagation axis}

The total phase advance of the emitted field at the harmonic frequency is locked to the phase of the fundamental field and, in absence of dispersion effects, can be approximated as [2]

$$
\phi_{\text {tot }}=q \phi_{f}+\alpha I,
$$

where $q$ is the harmonic order and $\phi_{f}$ is the phase of the fundamental due to focusing. (As shown below in Sec. III D, dispersion effects do not play a significant role and we do not consider them in our analysis.) The second term describes the intensity-dependent atomic phase [11]. $I$ is the intensity of the fundamental field and $\alpha$ is the phase coefficient of the main trajectory contributing to harmonic generation $(\alpha=24$ $\left.\times 10^{-14} \mathrm{~cm}^{2} / \mathrm{W}[12]\right)$. Both the geometrical $\left(q \phi_{f}\right)$ and the atomic $(\alpha I)$ phase shifts are important. In order to obtain good phase matching, the variation of the total phase $\phi_{t o t}$ should be as small as possible. This can be achieved if both the phase and intensity of the fundamental field are constant over the length of the interaction region or if they compensate each other so that $\phi_{t o t}$ is constant [11].

\section{Two foci vs one focus}

Figure 2 clarifies how phase matching along the propagation axis can be improved by using two foci instead of one. Figure 2(a) shows the phase of the fundamental beam along the propagation axis for two single-focus beams (focal points located at $z=0$ and $z=6.2 \mathrm{~mm}$ ) and a two-foci beam, generated as described in Sec. II, with the same focal points. The corresponding intensity variations are shown in Fig. 2(b). For all the calculations presented below, the spot size of the beam before the aperture is $40 \mathrm{~mm}$, the aperture diameter is $12 \mathrm{~mm}$, the pulse duration is $150 \mathrm{fs}$, and the energy $1.5 \mathrm{~mJ}$. For the two-foci beam, the phase factor between the two polarization components is chosen to be $\phi=4.8 \mathrm{rad}$. Clearly, it is possible to obtain a much slower phase and intensity change of the fundamental field in the two-foci case than in the one-focus case. These conditions should therefore lead to improved phase matching on axis compared to a setup with only one focus. 

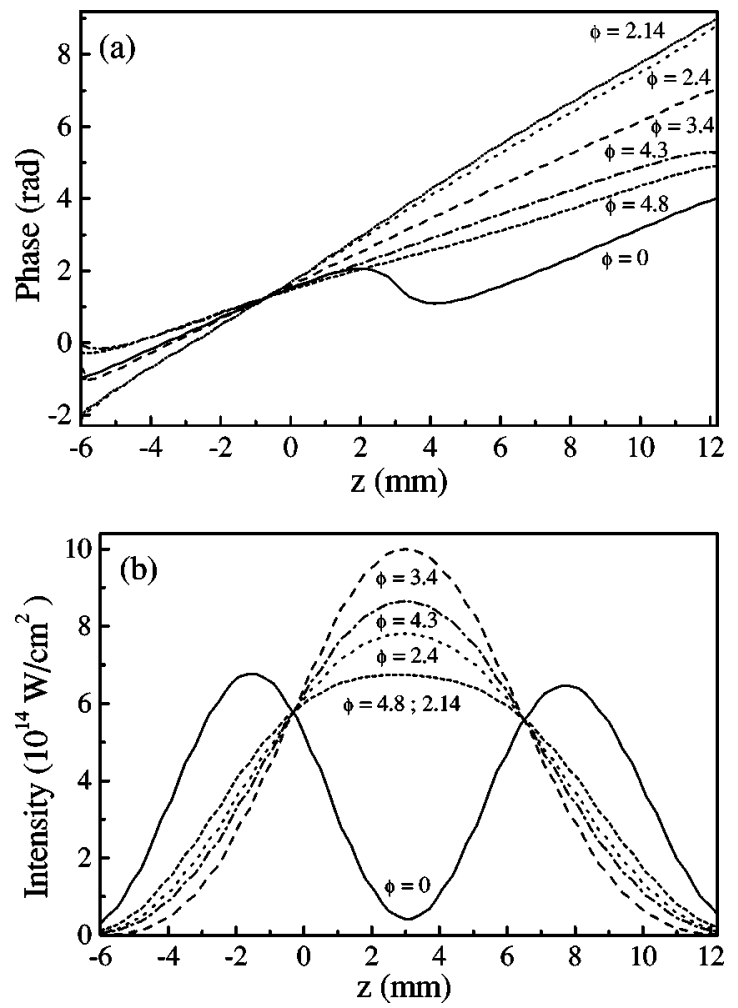

FIG. 3. Phase (a) and intensity profile (b) of the total fundamental field in the focal region on axis in the two-foci configuration. The phase differences $\phi$ between the two foci are 0 rad (solid line), $2.14 \mathrm{rad}$ (short dotted line), $4.8 \mathrm{rad}$ (short dashed line), $2.4 \mathrm{rad}$ (dotted line), $4.3 \mathrm{rad}$ (dot-dashed line), and $3.4 \mathrm{rad}$ (dashed line).

\section{Role of the phase difference between the two polarization components}

One advantage of using a two-foci setup is the additional degree of freedom presented by the phase difference $\phi$. This allows us to control phase matching on axis by tuning $\phi$. As discussed above, it is preferable to have a field with high and flat intensity and a flat phase along the propagation axis in order to obtain a high number of harmonic photons. However, the phase and intensity variations along the propagation axis are locked and it is difficult to fulfill these conditions simultaneously.

In Fig. 3, we show the phase (a) and intensity (b) variations of the fundamental field along the propagation axis for six different values of $\phi$. For $\phi=3.4$ the intensity is the highest possible, but the fundamental phase has a rather steep slope. For $\phi=4.8$ and $\phi=2.14$, the intensity is very flat over a long range, but the phase of the fundamental looks completely different, with a steep slope for $\phi=2.14$ and a very flat slope for $\phi=4.8$. The fundamental fields with $\phi$ $=2.4$ and $\phi=4.3$ have intermediate intensities; again the phase variation is much slower for one of them, $\phi=4.3$. For $\phi=0$ it is even possible to obtain a negative slope of the fundamental phase (around $z=3.1 \mathrm{~mm}$ ) which could possibly compensate for dispersion due to free electrons (leading to a positive slope of the fundamental phase). However, in the region where the phase has a negative slope, the intensity is low.

Also, the transverse intensity variation and, hence, the volume where harmonics can be generated depend on $\phi$.

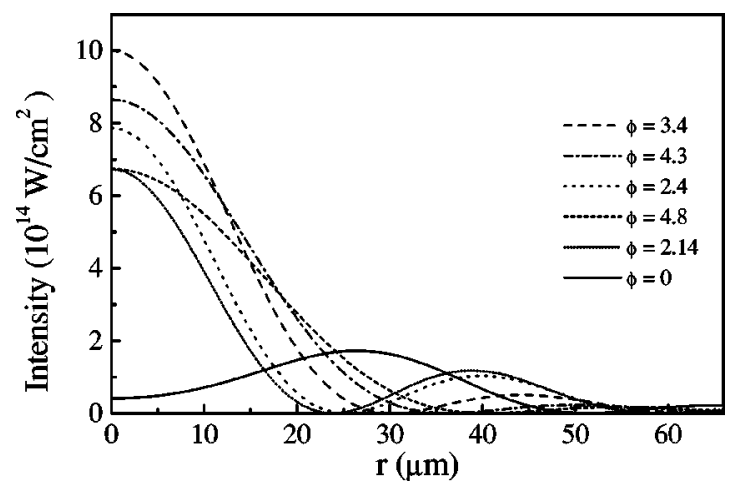

FIG. 4. Transverse intensity variation of the total fundamental field in the two-foci configuration at $z=3.1 \mathrm{~mm}$ (in the middle between the two foci). The phase differences $\phi$ between the two foci are $0 \mathrm{rad}$ (solid line), $2.14 \mathrm{rad}$ (short dotted line), $4.8 \mathrm{rad}$ (short dashed line), $2.4 \mathrm{rad}$ (dotted line), $4.3 \mathrm{rad}$ (dot-dashed line), and 3.4 rad (dashed line).

Figure 4 shows the transverse intensity profile in the middle of the two foci, $z=3.1$, for the same set of $\phi$ 's as in Fig. 3 . We see that for the highest peak intensities $(\phi=3.4, \phi=4.3)$ the spot size is quite small. It is also interesting to note the large difference in spot size for the beams with phases $\phi=4.8$ and $\phi=2.14$ though they have the same peak intensity and intensity variation along the propagation axis.

In summary, the best conditions for a high harmonic efficiency are a high intensity in a large volume (to optimize generation), together with a flat intensity profile and a slow phase variation along the propagation axis (to optimize phase matching). Since these requirements are usually not fulfilled at the same time, the harmonic efficiency is optimized by finding "good compromises."

These one-dimensional considerations of phase matching on the propagation axis illustrate, in a simple way, the idea behind our work. In order to get a more complete picture of phase matching, however, it is important to consider what happens in the entire volume of harmonic emission. To this end we use the graphical technique presented in [12].

\section{Phase-matching maps}

Let us briefly describe the method used to represent in space the phase-matching conditions [12]. The wave vector $\mathbf{k}_{p o l}$ of the polarization at position $(r, z)$ is (we consider $k$ $=2 \pi / \lambda$ and neglect dispersion; see Sec. III D)

$$
\mathbf{k}_{p o l}=\nabla\left[q k z+q \phi_{f}(r, z)+\alpha I(r, z)\right]=q \mathbf{k}_{1}+\mathbf{K},
$$

where $\mathbf{k}_{1}$ is the total wave vector of the fundamental beam and $\mathbf{K}=\boldsymbol{\nabla}[\alpha I(r, z)]$ is the atomic wave vector. The length of the wave vector $\mathbf{k}_{q}$ of the $q$ th harmonic field can be approximated as $\left|\mathbf{k}_{q}\right|=2 \pi q / \lambda$. In the ideal case, $\mathbf{k}_{q}=\mathbf{k}_{\text {pol }}$. The wave vector mismatch is defined as

$$
\delta \mathbf{k}=\mathbf{k}_{q}-q \mathbf{k}_{1}-\mathbf{K} .
$$

We define the coherence length $L_{c o h}$ as

$$
L_{c o h}=\frac{\pi}{|\delta \mathbf{k}|} .
$$



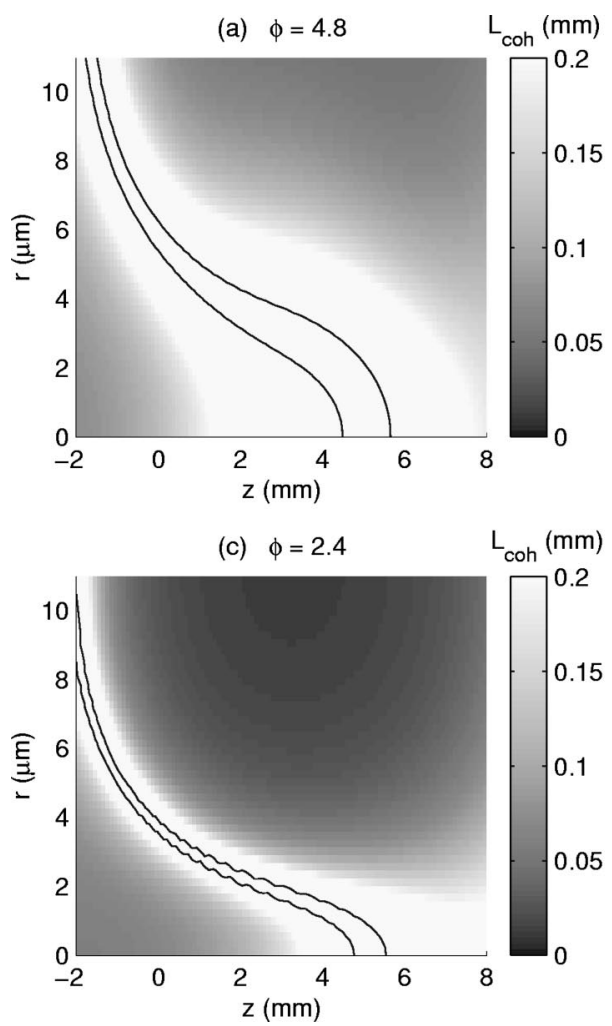
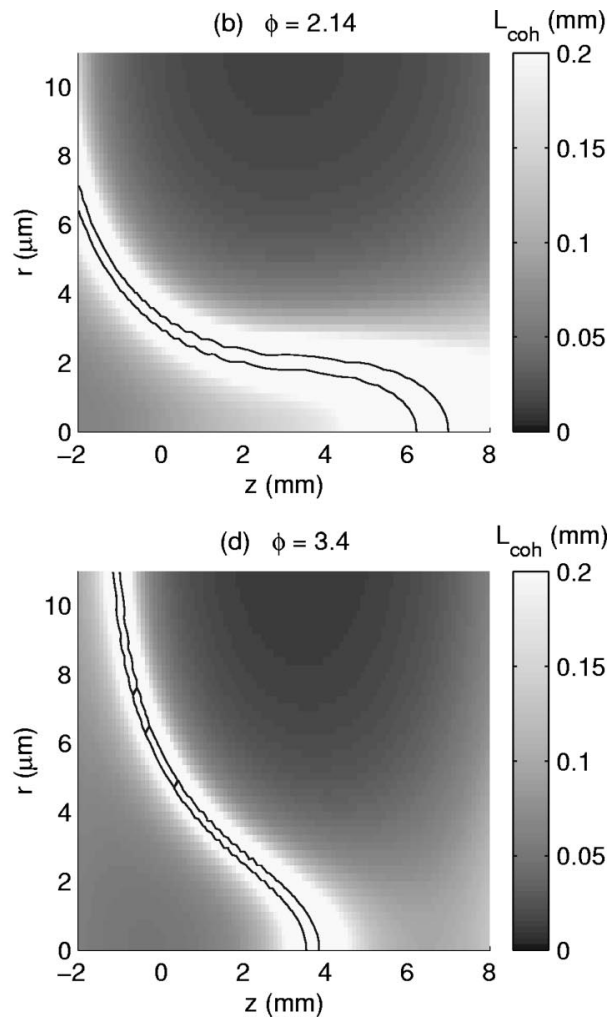

FIG. 5. Phase-matching maps for different values of the phase difference between the two foci, $\phi$. In (a) $\phi=4.8$, in (b) $\phi$ $=2.14$, in (c) $\phi=2.4$, and in (d) $\phi=3.4$. The coherence length $L_{c o h}$ is plotted on a gray-level scale according to the color bars to the right of the figures. Note that in the white-colored areas, the coherence lengths can be longer than the maximum color bar value $(0.2 \mathrm{~mm})$. Inside the black contour line, the coherence length is longer than $1 \mathrm{~mm}$. The phasematching maps reflect the situation for the 33rd harmonic, a pulse energy of $1.5 \mathrm{~mJ}$, a pulse duration of $150 \mathrm{fs}$, and an aperture diameter of $12 \mathrm{~mm}$.
In the phase-matching maps we plot $L_{c o h}$ on a gray-level scale where white means long coherence length (good phase matching) and black means short coherence length (poor phase matching). The scale is indicated by the color bars to the right of the plots. Note that in the white areas the coherence length can be longer than the maximum value given in the color bar.

In Fig. 5 we show phase-matching maps in the focal region for $\phi=4.8$ (a), $\phi=2.14$ (b), $\phi=2.4$ (c), and $\phi=3.4$ (d) for the 33rd harmonic. There are large differences in the coherence lengths between these four cases.

Inside the black contour lines in Fig. 5 the coherence length is above $1 \mathrm{~mm}$ which is the length of the interacting medium. The length of the region with $L_{c o h} \geqslant 1 \mathrm{~mm}$ on axis varies from $0.3 \mathrm{~mm}(\phi=3.4)$ to $1.2 \mathrm{~mm}(\phi=4.8)$. The volume where phase matching is good is much larger for $\phi$ $=4.8$ than for the other values of $\phi$.

In Fig. 6, we represent in a similar way the intensity distribution of the fundamental field for the same values of $\phi$. The interesting region, above the cutoff intensity for the 33rd harmonic, is indicated with a black contour line. The volume in which harmonics can be generated is largest for $\phi=4.8$ [Fig. 6(a)] though the peak intensity is not so high. For $\phi$ $=3.4[$ Fig. 6(d)], with the highest peak intensity, the generating volume is smaller.

When the peak intensity is high and the beam waist small, the atomic phase varies significantly in space, and phase matching becomes harder to achieve. It is also interesting to compare these plots with those for the one-focus case (Fig. 7) obtained in the same conditions (i.e., same aperture diameter, energy, and harmonic order). Here, the length of the region with $L_{c o h} \geqslant 1 \mathrm{~mm}$ on axis is only $0.15 \mathrm{~mm}$. It is clear that it is possible to get much better phase matching in the two-foci configuration both over a long range on axis and also in a larger volume.

\section{Dispersion effects}

We here briefly investigate the influence of dispersion due to neutral atoms and to free electrons by estimating the coherence length for these two effects and compare with the coherence length limited by the atomic phase and the focusing found in the previous section.

Let us first consider the coherence length caused by dispersion due to the neutral atoms, as defined in Eq. (7), $L_{c o h_{N A}}=\pi /\left|\delta \mathbf{k}_{N A}\right|$, where $\delta \mathbf{k}_{N A}=\mathbf{k}_{N A_{q}}-q \mathbf{k}_{N A_{1}}=(2 \pi q / \lambda)$ $\times\left(n_{q}-n_{0}\right)$. Here $n_{q}$ and $n_{0}$ are the refractive indices at the harmonic and laser wavelengths, respectively $[13,14]$. For an estimated pressure of 15 mbar, for the 33rd harmonic, and neglecting ionization we get a coherence length due to neutral atoms of $L_{\mathrm{coh}_{N A}}=13 \mathrm{~mm}$. This coherence length is clearly much longer than the ones obtained in Sec. III C and the influence of neutral atoms is thus negligible.

The effect of free electrons can be calculated in a similar way. At the intensities estimated in the medium, 4-6 $\times 10^{14} \mathrm{~W} / \mathrm{cm}^{2}$, less than $15 \%$ of the neon atoms are ionized which leads to a lower limit of the coherence length of $1 \mathrm{~mm}$. The presence of free electrons might in some cases reduce the area of good phase matching (Sec. III C) but not in a significantly manner.

\section{EXPERIMENTAL STUDY AND RESULTS}

First, we compare the maximum number of harmonic photons obtained in the two-foci setup with that obtained in a single focus configuration. Then we investigate how different values of the phase difference $(\phi)$ between the two polarizations and hence the intensity and phase variation of the fundamental field in space influence the harmonic signal. Finally, we attempt to optimize one specific harmonic and change the aspect of the harmonic spectra. 
(a) $\phi=4.8 \quad$ Int. $\left(10^{14} \mathrm{~W} / \mathrm{cm}^{2}\right)$

(b) $\phi=2.14 \quad$ Int. $\left(10^{14} \mathrm{~W} / \mathrm{cm}^{2}\right)$

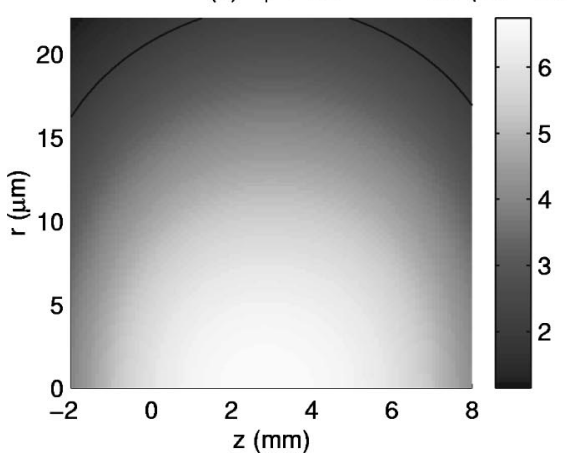

(c) $\phi=2.4$ nt. $\left(10^{14} \mathrm{~W} / \mathrm{cm}^{2}\right)$

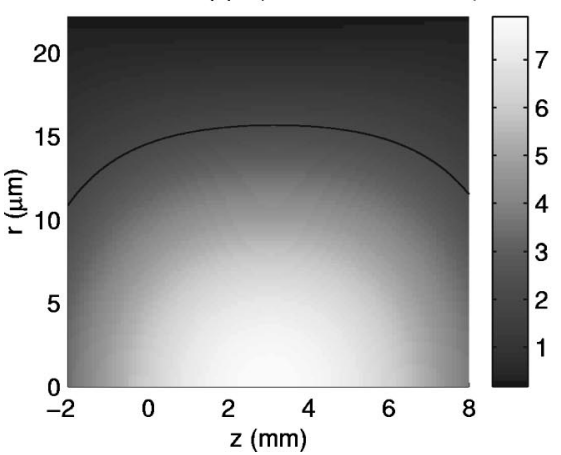

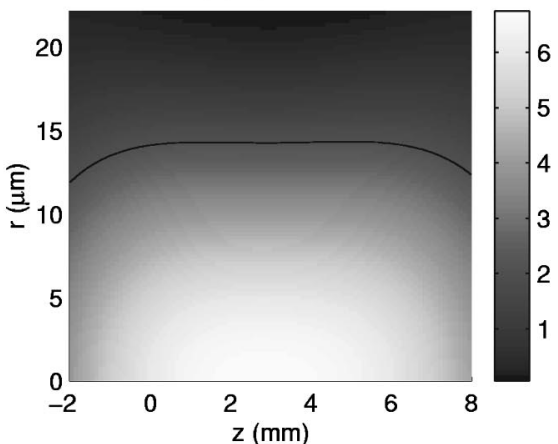

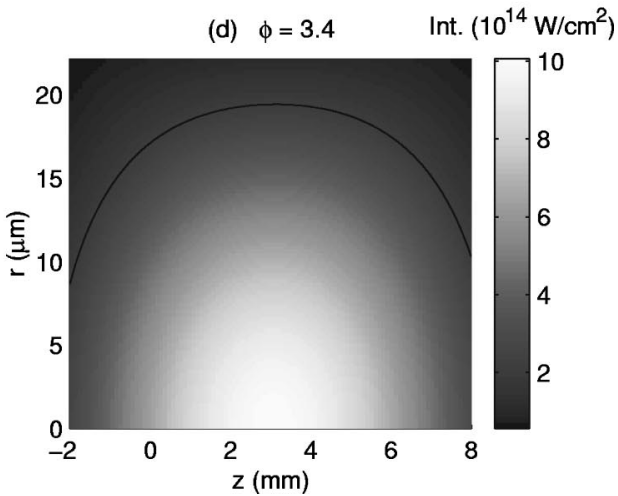

FIG. 6. Intensity maps for different values of the phase difference between the two foci, $\phi$. In (a) $\phi=4.8$, in (b) $\phi=2.14$, in (c) $\phi=2.4$, and in (d) $\phi=3.4$. The maps correspond to the phasematching maps shown in Fig. 5. The intensities can be read off the color bars to the right of the figures. The contour line shows the cutoff intensity for the 33rd harmonic. The conditions are the same as in Fig. 5.

\section{A. Comparison between the conversion efficiency in the one-focus and two-foci cases}

In order to compare the best case scenarios in the oneand two-foci configurations, all variable parameters are adjusted to get the highest possible signal. In each case we optimize the total beam energy, the aperture diameter, the phase difference between the two foci, and the gas-jet posi- tion relative to the foci. We show the harmonic spectra obtained in neon in Fig. 8. In the two-foci case (solid line) the aperture is $12 \mathrm{~mm}$, the energy in the interaction region is 5 $\mathrm{mJ}$, the phase difference between the two beams is $5 \mathrm{rad}$, and the gas jet is positioned $4 \mathrm{~mm}$ after the first focus.

A typical plateau harmonic in the two-foci case has an energy of $0.15-0.2 \mathrm{~nJ}$, corresponding to a conversion effi-

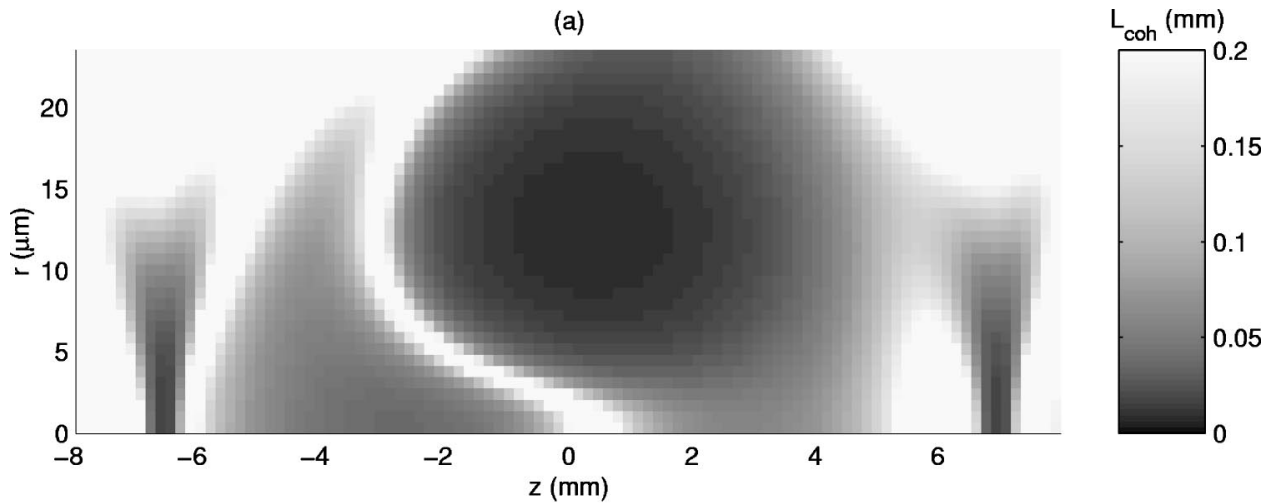

(b)

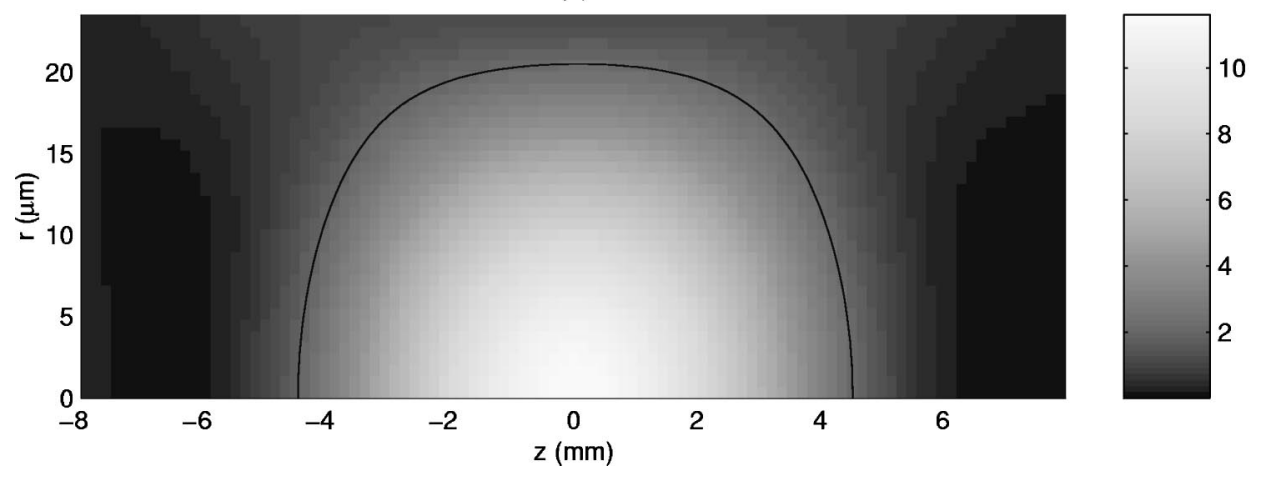

FIG. 7. Phase-matching map for the 33rd harmonic (a) and intensity map (b) for the one-focus configuration. In the phasematching map the coherence length is given in $\mathrm{mm}$ on the color-bar scale and in the intensity map the intensity is given in $\mathrm{W} / \mathrm{cm}^{2}$. Same conditions as in Figs 5 and 6. 


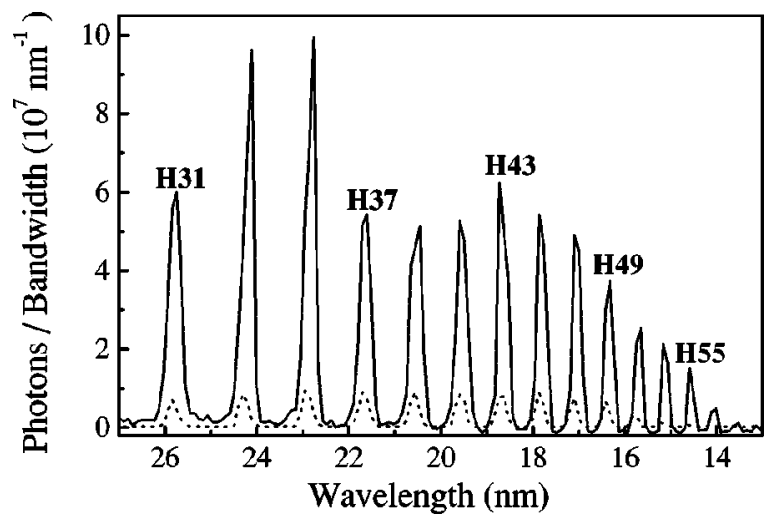

FIG. 8. Comparison between harmonic spectra in the two-foci case (solid line) and the one-focus case (dotted line). In the two-foci case the pulse energy is $5 \mathrm{~mJ}$, the aperture diameter $12 \mathrm{~mm}$, and the phase difference $\phi=5 \mathrm{rad}$. In the one-focus case the energy is 3 $\mathrm{mJ}$ and the aperture diameter $13 \mathrm{~mm}$.

ciency (in energy) of $3 \times 10^{-8}$. In the one-focus case (dotted line), the aperture is $13 \mathrm{~mm}$, the energy after the polarizer is $3 \mathrm{~mJ}$, and the gas jet is $4.5 \mathrm{~mm}$ after the focus. At this position, the intensity in the jet is estimated to be considerably lower than in the two-foci case. In the one-focus case, a typical harmonic has an energy of $0.02 \mathrm{~nJ}$, corresponding to a conversion efficiency of $7 \times 10^{-9}$. The peak intensity (highest intensity obtained anywhere in space) is the same in both cases.

In order to understand the difference in conversion efficiency of a factor of 4 , we compare the phase-matching maps for the one-focus case [Fig. 7(a)] and the two-foci case [Fig. $5(\mathrm{a}), \phi=4.8]$. In the one-focus case, phase matching on axis is good only around $z=0$. The coherence length is longer than $1 \mathrm{~mm}$ only in an interval of $0.15 \mathrm{~mm}$ in the direction of propagation. Transverse to the axis, phase matching rapidly becomes very poor and the emitting volume is small. Consequently, the highest possible signal is not obtained when the medium is close to focus, but farther out around $z$ $=4.5 \mathrm{~mm}$, where the phase-matching conditions are somewhat better. At this position the intensity is, however, rather low. The harmonic signal as a function of gas jet position is therefore low everywhere in space, limited by either intensity or coherence length or both.

In the two-foci case, on the other hand, there is a large region between the two foci, both along the propagation axis and transverse to it, that presents rather good phase-matching conditions. The volume in which good phase matching can be obtained is much larger than in the one-focus case. The intensity is also quite high in this area [Fig. 6(a)]. The advantage of the two-foci case is thus that it is possible to work with a high intensity and still get good phase matching in a large volume.

One of the drawbacks with our present setup is that half or more of the beam energy is reflected by the polarizer and thereby wasted. Although using half of the beam energy can have experimental advantages, the natural question to ask is what harmonic signal would be obtained if we could use all the energy in the beam. We have therefore performed a test in which we remove the polarizer and the birefringent compensator and use only one (linearly polarized) focus and thereby get access to the entire beam with an energy of $9 \mathrm{~mJ}$.

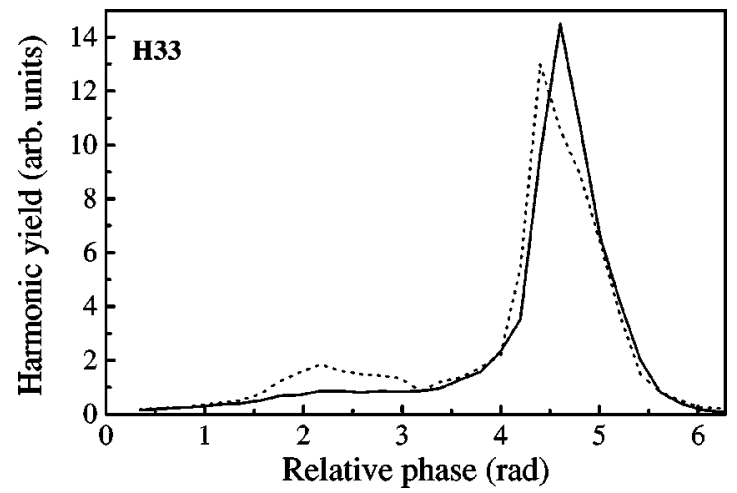

FIG. 9. Harmonic yield versus phase difference $\phi$ between the two orthogonally polarized fields in the two-foci case. The aperture diameter is $12 \mathrm{~mm}$ and the gas-jet position is $4 \mathrm{~mm}$ after the first focus. The energy in the interacting region is $4 \mathrm{~mJ}$ (solid line) and $5 \mathrm{~mJ}$ (dashed line).

We optimize the focusing conditions by inserting an aperture of $13 \mathrm{~mm}$ in the beam. The harmonic signal we obtain in these conditions is similar to that obtained with two foci (using half the beam energy). In this respect, the conversion efficiency in the two-foci case only increases with a factor of 2 compared to the one-focus case using all the energy in the beam.

\section{B. Influence of phase difference on the harmonic efficiency}

In Fig. 9 we show the 33rd harmonic in neon as a function of the phase difference $\phi$ between the two orthogonally polarized fields for two different energies. The gas jet is located in between the two foci, $4 \mathrm{~mm}$ after the first focus. The total energy transmitted through the polarizer (4 and $5 \mathrm{~mJ}$, respectively) is kept constant. [The aperture is large enough (12.3 $\mathrm{mm}$ ) to make the transmitted energy independent of $\phi$.] The 33rd harmonic signal is measured directly after the slit at a fixed wavelength of $24.2 \mathrm{~nm}$. Figure 9 shows that the harmonic signal is strongly dependent on $\phi$. $\phi$ is determined experimentally by measuring the energy transmitted through an aperture as one of the birefringent wedges is translated. This is repeated for various diameters of apertures and fitted to calculations. We have checked that signal $(\phi)$ $=\operatorname{signal}(\phi+2 \pi)$. There is a clear maximum at $\phi$ $=4.6 \mathrm{rad}$, and for the highest energy, there is also a small maximum around $\phi=2.2 \mathrm{rad}$.

These values agree remarkably well with the predictions of Secs. III B 2 and III C. At $\phi=4.8$ (this compares well to the experimental value $\phi=4.6$ ), we have the largest generating volume together with good phase-matching conditions. At $\phi=2.14$ (experimentally, $\phi=2.2$ ), the intensity on axis is relatively high, but the phase-matching conditions are much worse and the generating volume is also somewhat smaller. These maxima are a clear effect of the good phase-matching conditions in a large volume.

\section{Control results}

An additional advantage offered by the two-foci technique is the possibility of control. In Fig. 10 we show two curves, one obtained with two foci (phase difference $4.3 \mathrm{rad}$ between the two polarizations) and the gas jet placed $4 \mathrm{~mm}$ after the first focus, the other with one focus and gas-jet 2 


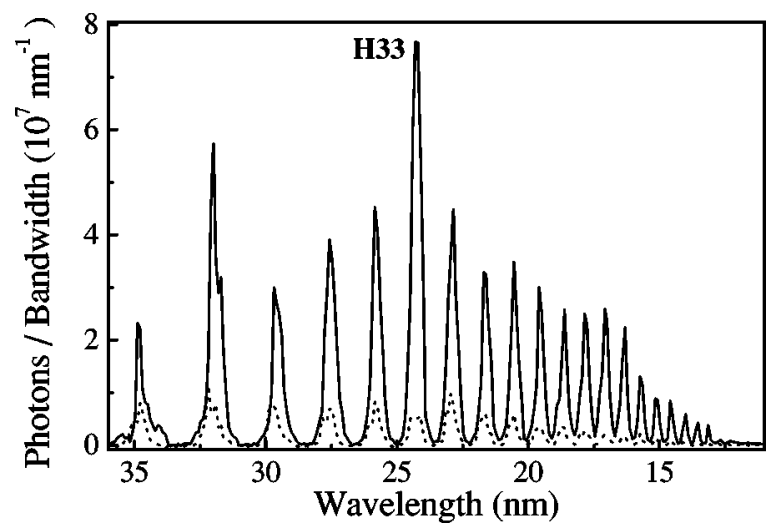

FIG. 10. Harmonic spectra optimized for the 33rd harmonic. The solid line shows the two-foci case and the dotted line shows the one-focus case. The energy is $4 \mathrm{~mJ}$ and the aperture is $12 \mathrm{~mm}$ in both cases. The phase difference $\phi$ in the two-foci case is $4.3 \mathrm{rad}$.

$\mathrm{mm}$ before focus. The aperture is $12 \mathrm{~mm}$ and the input energy is $4 \mathrm{~mJ}$ in both cases. The intensities at the gas-jet positions are the same in the two cases. The result is obtained by optimizing specifically all the parameters (including the phase difference $\phi$ ) for the 33rd harmonic. The average number of photons in one of the harmonic peaks, except the $33 \mathrm{rd}$, in the two-foci case is $1-1.5 \times 10^{7}$ (energy $0.1 \mathrm{~nJ}$ ) compared to $2 \times 10^{6}$ photons (energy $0.02 \mathrm{~nJ}$ ) in the one-focus case. The 33 rd harmonic contains $4 \times 10^{7}$ photons in the two-foci case with an energy of $0.3 \mathrm{~nJ}$. The conversion efficiency for the $33 \mathrm{rd}$ harmonic is $7 \times 10^{-8}$, i.e., almost 3 times the conversion efficiency for the adjacent harmonics $\left(2.5 \times 10^{-8}\right)$. The energy in the $33 \mathrm{rd}$ harmonic seems really to increase on behalf of the others since the energy in these peaks is lower than in the results presented above.

In Fig. 11, we study the harmonic spectra for different

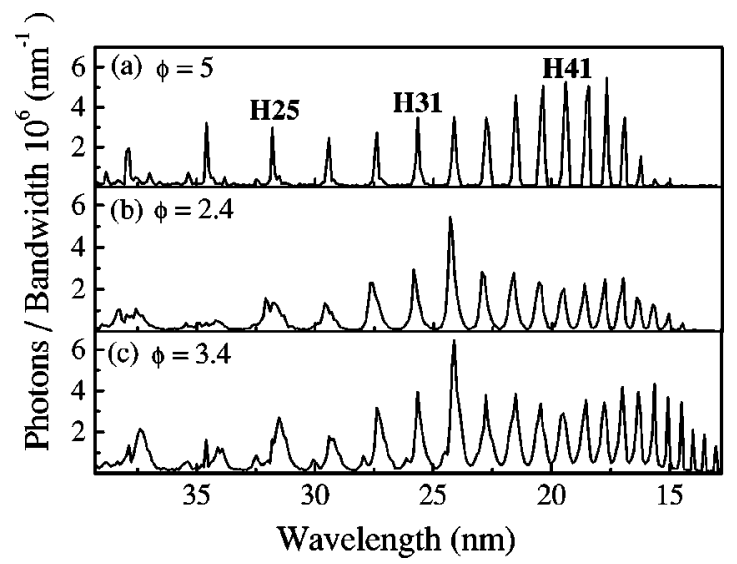

FIG. 11. Harmonic spectra for three different phase differences $\phi$ between the two polarizations in the two-foci case. The energy in the interacting region is $2 \mathrm{~mJ}$, the aperture diameter is $12 \mathrm{~mm}$ and the gas jet is positioned $3.5 \mathrm{~mm}$ after the first focus in all three cases. In (a) the phase difference on axis is $5 \mathrm{rad}$, in (b) $2.4 \mathrm{rad}$, and in (c) $3.4 \mathrm{rad}$. values of the phase difference between the two polarizations. These spectra are obtained with an energy of $2 \mathrm{~mJ}$, an aperture of $12 \mathrm{~mm}$, and with the gas jet positioned $3.5 \mathrm{~mm}$ after the first focus. The phase difference is $5 \mathrm{rad}$ in (a), $2.4 \mathrm{rad}$ in (b), and $3.4 \mathrm{rad}$ in (c). There are quite a few differences in these spectra. First, the spectral widths of the harmonic peaks change significantly. For the 31 st harmonic, for example, the width at half maximum is $0.12 \mathrm{~nm}$ in (a), $0.25 \mathrm{~nm}$ in (b), and $0.28 \mathrm{~nm}$ in (c). Second, the shapes of the plateaus are quite different. In (a) the number of photons in the harmonic peaks increases towards higher frequencies whereas in (b) and (c) it decreases. The number of photons in the 41st harmonic is $1 \times 10^{6}$ in (a), $0.6 \times 10^{6}$ in (b), and $0.2 \times 10^{6}$ in (c). For the 25th harmonic we have $0.3 \times 10^{6}$ photons in (a), $1.2 \times 10^{6}$ in (b), and $1.5 \times 10^{6}$ in (c).

To interpret this spectra, we consider the phase-matching maps and the intensity maps for $\phi=4.8$ (a), $\phi=2.4$ (c), and $\phi=3.4$ (d) in Figs. 5 and 6. The narrow peaks in (a) are thus obtained with a long coherence length and low intensity (4 $\left.\times 10^{14} \mathrm{~W} / \mathrm{cm}^{2}\right)$ in a large generating volume. The broader peaks in (b) and (c) are obtained with shorter coherence lengths, higher intensity $\left(5 \times 10^{14}\right.$ and $\left.6 \times 10^{14} \mathrm{~W} / \mathrm{cm}^{2}\right)$ respectively, and a smaller generating volume. The significant difference in width between the harmonics in (a) and those in (b) or (c) could be related to the difference in intensity. It could also indicate that different quantum paths [11] are involved in the generation of these harmonics: the short quantum path $\left(\tau_{1}\right)$, leading to a narrow spectral width in (a), and the longer quantum path ( $\tau_{2}$, often dominant), leading to a broader spectrum in (b) and (c).

\section{SUMMARY}

In this proof of feasibility experiment, we have shown that it is possible to increase the conversion efficiency for harmonic generation in neon with a factor of 4 by using a fundamental beam with two foci along the propagation axis insted of one. This comparison is done with similar focusing conditions, i.e., an apertured beam focused by the same lens. The harmonic signal is strongly dependent on the phase difference between the two components focused into the two foci. It is possible to explain this variation by considering the complicated interplay between phase matching, intensity, and volume effects. A spin-off result of this experiment is the ability to increase the conversion efficiency of one single harmonic on behalf of the others and to control the shape of the plateau and the widths of the harmonic peaks by changing the phase difference between the foci.

\section{ACKNOWLEDGMENTS}

Support from the Swedish Natural Science Research Council and from the EC's Access to Large Scale Facilities' Program (Contract No. ERBFMGECT950020) is acknowledged. We thank Philippe Antoine for stimulating discussions at the beginning of this work.
[1] L.A. Lompré, A. L'Huillier, M. Ferray, P. Monot, G. Mainfray and C. Manus, J. Opt. Soc. Am. B 7, 754 (1990).

[2] P. Salières, A. L'Huillier, and M. Lewenstein, Phys. Rev. Lett. 74, 3776 (1995).
[3] M. Schnürer, Z. Cheng, S. Sartania, M. Hentschel, G. Tempea, T. Brabec, and F. Krausz, Appl. Phys. B: Lasers Opt. 67, 263 (1998).

[4] Andy Rundquist, Charles G. Durfee III, Zenghu Chang, Cathe- 
rine Herne, Sterling Backus, Margaret M. Murnane, and Henry C. Kapteyn, Science 280, 1412 (1998).

[5] E. Constant, D. Garzella, P. Breger, E. Mével, Ch. Dorrer, C. Le Blanc, F. Salin, and P. Agostini, Phys. Rev. Lett. 82, 1668 (1999).

[6] Yusuke Tamaki, Jiro Itatani, Yutaka Nagata, Minouru Obara, and Katsumi Midorikawa, Phys. Rev. Lett. 82, 1422 (1999).

[7] I. Mercer, E. Mével, R. Zerne, A. L'Huillier, P. Antoine, and C.-G. Wahlström, Phys. Rev. Lett. 77, 1731 (1996).

[8] S. Svanberg, J. Larsson, A. Persson, and C.-G. Wahlström, Phys. Scr. 49, 187 (1994).
[9] C.-G. Wahlström, J. Larsson, A. Persson, T. Starczewski, S. Svanberg, P. Salières, Ph. Balcou, and Anne L'Huillier, Phys. Rev. A 48, 4709 (1993).

[10] A. E. Siegman, Lasers (University Science Books, Mill Valley, CA (1986).

[11] M. Lewenstein, P. Salières, and A. L'Huillier, Phys. Rev. A 52, 4747 (1995).

[12] Ph. Balcou, P. Salières, A. L'Huillier and M. Lewenstein, Phys. Rev. A 55, 3204 (1997).

[13] P. J. Leonard, At. Data Nucl. Data Tables 14, 21 (1974).

[14] B. L. Henke, E. M. Gullikson, and J. C. Davis, At. Data Nucl. Data Tables 54, 181 (1993). 